This item was submitted to Loughborough's Research Repository by the author.

Items in Figshare are protected by copyright, with all rights reserved, unless otherwise indicated.

\title{
The user experience of crowds: a human factors challenge
}

PLEASE CITE THE PUBLISHED VERSION

https://www.hfes.org//Publications/

\section{PUBLISHER}

(c) Human Factors and Ergonomics Society

VERSION

AM (Accepted Manuscript)

LICENCE

CC BY-NC-ND 4.0

REPOSITORY RECORD

Kendrick, Victoria L., and Roger Haslam. 2019. "The User Experience of Crowds: A Human Factors Challenge". figshare. https://hdl.handle.net/2134/12497. 
This item was submitted to Loughborough's Institutional Repository (https://dspace.lboro.ac.uk/) by the author and is made available under the following Creative Commons Licence conditions.

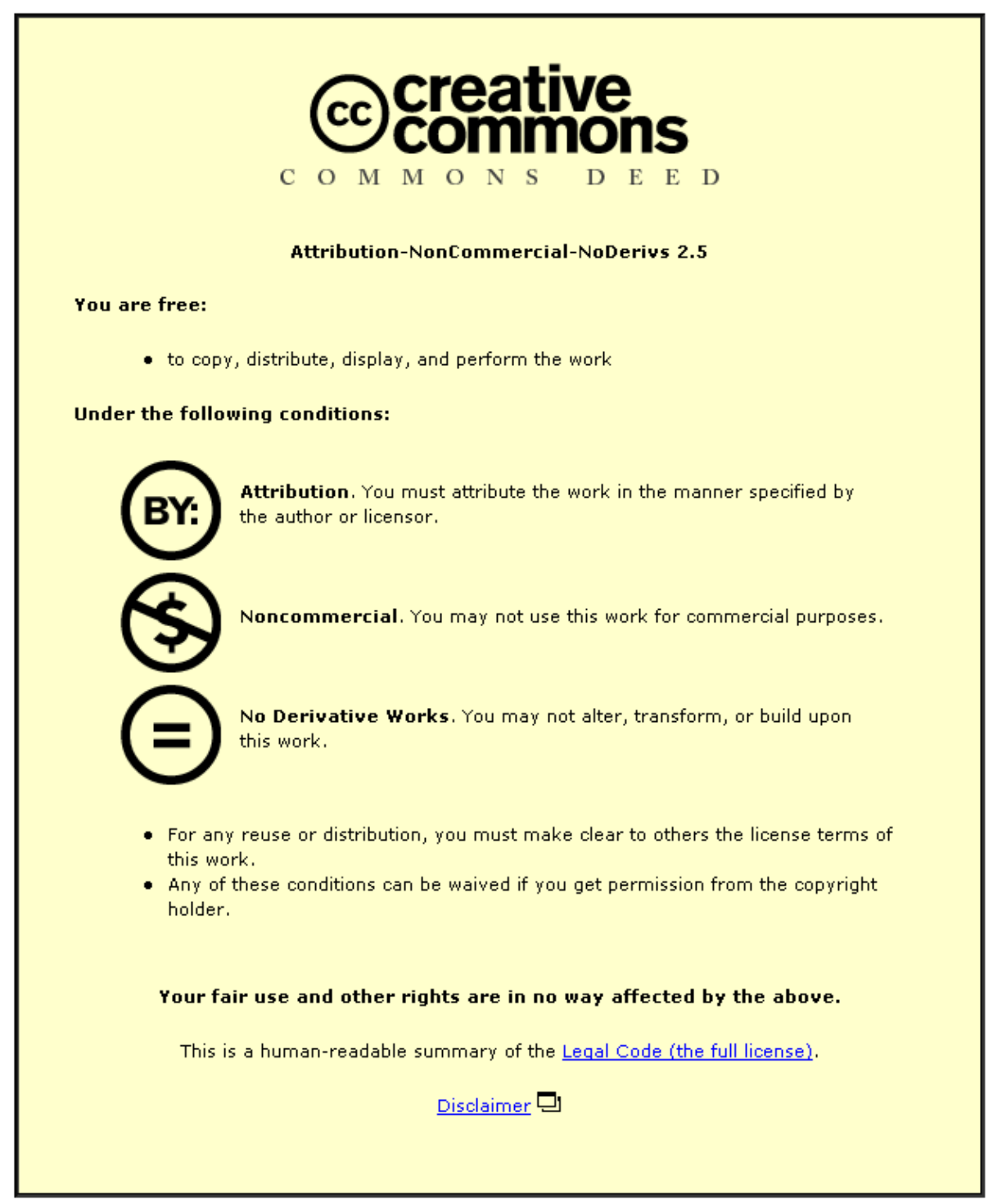

For the full text of this licence, please go to: http://creativecommons.org/licenses/by-nc-nd/2.5/ 


\title{
The User Experience of Crowds - A Human Factors Challenge
}

\author{
Victoria L. Kendrick, Roger A. Haslam \\ Loughborough University, United Kingdom
}

\begin{abstract}
Crowds are such an integral part of our everyday lives, yet research considering how the user experience of crowds can be enhanced, remains relatively underdeveloped. As with any other user interaction, the key human factors considerations of user safety, performance, comfort and satisfaction apply. A review of literature highlighted that important factors influencing the operation of crowds have been studied relatively independently, tending to focus on specific crowd situations, but rarely accounting for the interaction of factors and similarities and differences between crowd types. Focus groups exploring individual experiences of crowds revealed differences and priorities varying with age and expectations. This initial study contributes towards modeling contributory factors to the crowd experience, moving towards a more holistic understanding.
\end{abstract}

\section{INTRODUCTION}

\section{Background Information}

Gatherings of people (hereafter referred to as crowds) are an everyday experience and it is surprising therefore that research concerning the overall crowd experience remains relatively underdeveloped. Substantial research has been carried out concerning specific factors affecting the crowd experience, including: satisfaction of individuals in crowds (Baum \& Greenberg, 1975; Altmans, 1975; Schmidt \& Keating, 1979; Machleit, Eroglu \& Mantel, 2000); performance of desired or necessary tasks (Klein \& Harris, 1979); individual personality (Worchel \& Teddie, 1976; Spacapan \& Cohen, 1983); psychological reactions to a given crowd situation (Worchel \& Yohai, 1979); prior expectations and experiences (Baum \& Greenberg, 1975; Webb \& Worchel, 1993) gender (Rustemli, 1992; Ozdemir, 2008) and culture (Pons, Laroche \& Mourali, 2006; Pons \& Laroche, 2007). Investigation has also focused on the contribution of different crowd situations to individual experience of stress (Cox, Houdmont \& Griffiths, 2006) and personal space preferences (Hasse \& Markey, 1973; Hayduk, 1983; Sinha \& Sinha, 1991; Rustemli et al., 1992; Kaya \& Erkip, 1999; Gerin-Lajoie, Richards \& McFadyen, 2005; Evans \& Wener, 2007; Martinez, 2009). Moreover, studies have considered a range of different crowd types, including: sporting events (Zhang, Liu, Wu \& Zhao, 2007; Johnson, 2008); retail environments (Machleit et al., 2000; Ozdemir, 2008; Whiting \& Nakos, 2008); religious pilgrimages (Hughes, 2002; 2003); restaurants (Tse, Sin \& Yim, 2002; Yildirim \& Akalin-Baskaya, 2007; Robson, 2008); and music festivals (Janchar, Samaddar \& Milzman, 2000). However, these investigations have tended to be uni-dimensional, focusing on single variables or particular crowd situations. The absence of research examining the combined contribution of factors to the overall crowd experience represents a gap in our knowledge.

\section{Goal of the Paper}

The existing literature is discussed, highlighting individual factors that have been identified as influential to the individual and collective user experience of crowds. Findings from focus groups are presented, providing insight into how these variables combine to affect the human factors considerations of user safety, performance, comfort and satisfaction, for different types of crowd involvement and different groups of people. Participants, all with a good level of independence and mobility, varied with regard to status, including international students, young professionals, parents of primary school children, healthy adults and older people, with a mix of gender, physical fitness and mobility. The groups considered different crowd situations, including: retail environments, religious events, transportation environments, tourism crowds and spectator events. These findings will contribute towards more holistic modeling of the user experience of crowds, identifying generic aspects and situation specific factors. Improved understanding in this respect should be to the ultimate advantage of both those who find themselves present in crowds and those responsible for organizing or overseeing gatherings.

\section{REVIEW}

Crowds are influenced by a variety of factors: satisfaction (how content individuals are in different types of crowds, and factors affecting the contentment of the collective); performance (the ability of the individual and collective to perform necessary or desired tasks within the crowd); and safety (the security of both individuals and the collective crowd). However, there is a distinct lack of concrete definitions relating to what constitutes a crowd and aspects of crowd behavior (Zeitz, Tan \& Zeitz, 2009).

\section{Psychological Dimensions of Crowding}

Almost forty years ago, Stokols (1972) suggested that density was a physical condition and crowding a psychological state. Stokols argued that the negative psychological experience of crowding results from interactions between environmental characteristics and personal factors, and not from high spatial density alone. Moreover, in 1976, Worchel and Teddie proposed that "it is not the amount of space available to the individual per se but the distance between individuals that determines the degree of stress arising from a particular situation". Thus, in the model proposed by Worchel and Teddie (1976), inappropriate closeness, rather than high density, was the necessary condition for an undesirable sense of crowding. Rustemli et al. (1992) suggested that for high density to produce crowding 
effects, spatial limitation must be the distinct feature of an environment. When confronted with inadequate space, a person has a reduced level of control over the situation and experiences stress and arousal that could lead to feelings of being crowded. Furthermore, Webb and Worchel (1993) suggested that high density could induce cognitive overload (Esser, 1973); impose behavioral constraints (Schopler \& Stockdale, 1977); evoke feelings of uncontrollability over one's environment (Rodin, Solomon \& Metcalf, 1978) and frustrate the goal of privacy (Altman \& Chemers, 1980), all of which could impact the experience of crowdedness. However, Freedman (1979) had already claimed that "virtually all of the active researchers began with the impression that crowding, defined here as physical density, is basically harmful to people". From a social psychology perspective, Worchel and Yohai (1979) widened the discussion, suggesting that several variables are associated with cognitive responses to crowding, including desires for privacy, density, territoriality, and control.

More recently, Yildirim et al. (2007) highlighted distinctions in researching the effects of crowding on human health and behavior. Primarily that crowding and close interpersonal distances increase stimulation, which may not be undesirable. However, some crowd situations can be threatening and foreboding, with extremes of stimulus intensity creating potentially stress inducing effects (Evans \& McCoy, 1998; Dion, 1999).

\section{Health and Emergency Medicine Research}

Arbon (2004) emphasized the contribution of health research for understanding the health effects of massgatherings. Arbon subsequently developed a conceptual model illustrating the relationship between: biomedical, environmental, and psychosocial factors at mass-gatherings, aiming to inform responses to emergency situations. However, a recent review of crowd behavior at mass gatherings with a similar focus, highlighted a distinct gap in theory and practice concerning crowd psychology in the mass-gathering setting (Zeitz et al., 2009).

\section{Methodology Used To Analyze Crowding}

In terms of methods used to study crowds, previous research has tended to focus on experimental techniques, which vary considerably with regard to ecological and external validity. Approaches have included: the presentation of photographs (Eroglu \& Machleit, 1990; Ozdemir, 2008), video recordings (Smith et al., 2009), or slides (Eroglu \& Machleit, 1990; Hui \& Bateson, 1991) of different crowd environments, accompanied by written crowd scenarios read to participants in order to imagine being in the situation under investigation (Pons et al., 2006). However, it is unclear how far such methodologies initiate valid and reliable responses compared with actual experiences of being in a crowd. To imagine oneself within a given type of crowd environment (for example a football stadium), does not provide sensory or social information gained through actually attending an event. Moreover, it is plausible that prior expectations and previous experiences of participating in a particular type of crowd situation might impact on the experience and satisfaction whilst in the crowd (Webb \& Worchel, 1993).

\section{Crowd Behavior}

Considerable attention has been devoted to the development of mathematical computer models, to replicate crowd flows and dynamics (Goldenstein et al., 2001; Musse \& Thalmann, 2001; Zhen et al., 2008; Smith et al., 2009). In 2008 , Zhen et al. utilized a new model to describe processes associated with crowd gatherings during disasters. The review focused on essential factors contributing to a severe trample disaster at the Lantern Festival of China, resulting in the death of 37 tourists. Quantitative post-event analysis was carried out using a mathematical model and simulation methods, highlighting descriptions of the whole process, factors contributing to the disaster, and characteristics of the trample.

Elsewhere, Lee and Hughes (2007) highlighted a distinct lack of research concerning the flow of large crowds of pedestrians associated with special events (sporting, music, and religious for example), against a preponderance of research into pedestrian flows in more routine walking environments.

With regard to predicting crowd behaviors, previous research suggests difficulties with this due to the irrational and erratic behavior of individuals in a crowd. There is other work however supporting the argument that human behavior is generally motivated by goals (Valach, Young \& Lynam, 2002; Lee \& Hughes, 2007). Thus, behavior in crowds is rational and predictable at the collective level. In summary, research into factors contributing to overall crowd experience is required, in order to understand the interaction of the variables and how the combination affects crowd experience.

\section{METHODOLOGY}

Five focus groups were utilized to gain insight and knowledge of the user experience of crowds, allowing flexibility to follow up interesting responses, and underlying motives. An initial focus group schedule was piloted, and amended, prior to programmed focus groups.

Photographs were presented to focus groups, in order to illicit discussion surrounding how individuals might feel within such crowd environments (Eroglu \& Machleit, 1990; Ozdemir, 2008). Crowd types investigated included: retail environments, religious events, transportation environments, tourism crowds and spectator events. Such an approach permitted respondents to discuss and comment on issues from their own perspective, within a group setting.

Participants in this study were recruited using a range of methods to achieve a structured convenience sample. Over a period of four months, emails were distributed to each of the five target focus groups: international students at University, young professionals (25-35 years), parents of young children, healthy adults (35-65 years), and older adults (over 65 years). This was to ensure that participants represented a range of individual circumstances and physical abilities. Each focus group comprised between six and eight individuals. Each focus group discussion was digitally recorded, with the knowledge and consent of participants. The same facilitator led each focus group, each lasting approximately 90 minutes.

Focus group recordings were subsequently transcribed verbatim, and imported into the qualitative software tool, NVivo (Version 8.0) to ensure systematic analysis. The qualitative analysis of focus group data was 
conducted following the three steps developed by Miles and Huberman (1994); data reduction, data display, and conclusion drawing and verification. Reliability was ensured through systematic review of the data by two researchers.

\section{RESULTS}

A total of 35 participants were involved (see Table 1: Focus group participant data). The age of participants ranged from 21-71 years (mean 39.5 years; standard deviation 17.0 years), with 15 males and 20 females.

\begin{tabular}{|l|c|c|c|c|}
\hline Focus Groups & $\begin{array}{c}\text { Number of } \\
\text { participants }\end{array}$ & $\begin{array}{c}\text { Age Range } \\
\text { (lowest - } \\
\text { highest) }\end{array}$ & $\begin{array}{c}\text { Mean } \\
\text { Age } \\
\text { (1 d.p.) }\end{array}$ & $\begin{array}{c}\text { Standard } \\
\text { Deviation } \\
\text { (1 d.p.) }\end{array}$ \\
\hline $\begin{array}{l}\text { International } \\
\text { students }\end{array}$ & 6 & $22-27$ & 25 & 1.7 \\
\hline $\begin{array}{l}\text { Young } \\
\text { professionals }\end{array}$ & 8 & $25-34$ & 27.1 & 3.2 \\
\hline $\begin{array}{l}\text { Parents of } \\
\text { young children }\end{array}$ & 6 & $21-32$ & 27.3 & 3.9 \\
\hline Healthy adults & 8 & $40-55$ & 47.3 & 4.3 \\
\hline Older adults & 7 & $65-71$ & 67.7 & 2.4 \\
\hline TOTAL & 35 & $21-71$ & 39.5 & 17.0 \\
\hline
\end{tabular}

Table 1: Focus group participant data.

\section{Emergent Themes}

Emergent themes (formulated from NVivo analysis of findings) are presented in order of frequency mentioned, with subsequent key differences highlighted.

Design and organization (sectioned areas, clear exit routes, seating, pedestrian flow system, view, toilet facilities). Participant 7: "The one good thing with supermarkets is that they have the signs up at the top to tell you where things are. So even if it's crowded and you don't know where something is, you can look up and find where to go." (Healthy adults, female aged 46 years)

Stress (anxiety, frustration, claustrophobia, vulnerability, intimidation). Participant 21: "See it would frustrate me if it was crowded, and you knew where your stuff was, but you couldn't get to it!" (Healthy adults - Retail crowding, male aged 40 years)

Safety and security (protection, slip, trip and fall hazards, trampling risks, violence). Participant 10: "Just having a steward in the crowd makes you feel a bit more reassured, to keep you safe or whatever." (Young adults, male aged 34 years)

Motivation (incentive to remain in the crowd, shared or enjoyable purpose, time constraints, financial motivation). Participant 3: "Because it's something that you want to do, you tolerate it at the time." (Older adults, female aged 65)

Mood (manners, boredom, hostility, excitement)

Environmental factors (weather, heat, vision, noise, pollution, odors). Participant 23: "I had a bad experience shopping in Nottingham city centre near to Christmas! There were just people everywhere... and it was really cold outside, so you've got your coat and scarf on, and then when you go into the shops its really hot and its horrible." (Healthy adults, male aged 47 years)

Movement (ability to move to required destination, disorientation, fear of losing people). Participant 7: "I'd panic in crowds! Especially if I went in with a friend and then lost them, that'd be horrible!" (Young adults, female aged 22)

Goal prevention (conflicting goals, prevention of goal achievement, competition between crowd members). Participant 9: "If you can't get to look at the thing that you want to look at.. cos there's somebody else in front of you.. and you're kind of waiting for them but they're being really slooooowww!" (Young professionals - Retail crowding, male aged 26 years)

Preconceptions (prior experience and expectations, cultural norms, stereotypes). Participant 3: "Also, with a lot of crowds you expect that there's going to be a lot of people there. So it's not going to be a surprise." (International students, female aged 26 years)

Behavior (inappropriate, antisocial, pushing)

Avoidance (of crowds, unavoidable crowds)

Space available (personal space)

Distractions (unfamiliar surroundings, factors that distract from the crowd situation). For example participant 30 said: "When there's things to look at in the crowd then it's not so bad. You're taking it all in, and it sort of occupies your mind." (Older adults - Tourism crowds, female aged 70 years) Control (feelings of uncontrollability, confusion)

Encumbrances (maneuvering trolleys, wheelchairs, push chairs, strollers, large bags, suitcases)

Company (accompaniment of friends, or feelings of loneliness in a crowd). Participant 6: "Sure, generally I prefer to be in a crowd with people that I know, so like close friends and things like that." (International students, male aged 25)

Atmosphere (positive and negative ambience)

Individual factors (physical height, age)

Communication (information available, language)

\section{Key Differences between Individual Groups}

Preconceptions: For older adults, prior expectations and familiarity with a situation appeared to contribute highly to overall crowd satisfaction, compared to the other groups involved. For example, participant 30 highlighted: "I mean that situation, in a supermarket you weren't familiar with... you'd be less happy than if it was your usual supermarket that you went to every week wouldn't you? Cos you're familiar with it." (Older adults, female aged 70)

Control: Feeling in control during crowd situations appeared to be particularly important to older individuals. For example, participant 35 suggested "Well.. if you've got your designated seat then it's ok.. but it's when you're standing up in a crowd and you've got less control of the situation that it's more of a problem." (Older adults, female aged 66 years)

Toilet facilities: The availability of toilet facilities, and seating were highlighted as influential to crowd satisfaction levels, for individuals over 65 years only, with no reference to this issue within any other group. For example, participant 30 stated that: "You see if you're in the middle of a crowd and you want to go to the toilet." (Older adults, female aged 70 years).

Encumbrances appear to strongly affect the crowd satisfaction of parents of young children. The navigation of push chairs through a crowd of people, or through supermarket aisles appeared to cause frustration. Participant 15 mentioned: "If you had a push chair you definitely 
wouldn't be able to get through a crowded street." (Parents of young children, female aged 25 years)

Company: Being accompanied by friends appeared to increase crowd satisfaction for international students and young professionals in particular. With participant 9 commenting: "When you're alone in the crowd it can be quite daunting." (Young professionals, male aged 26 years)

Violence was mentioned as an issue in the healthy adults group but not by any other age group. For example participant 25 suggested: "In a crowded pub, if somebody did lose it for whatever reason, there are plenty of things that they could use as a weapon if they wanted to." (Healthy adults, male aged 47 years)

Weather appeared to be an important environmental factor influencing crowd satisfaction of international students. Participant 2: "If the weather's nice everyone's sort of walking slowly, whereas if it is raining then everyone's going to be walking faster to get out of the rain. And rushing around and just like bumping into you, and it's just more of an unpleasant experience." (International student, female aged 22 years)

\section{DISCUSSION}

The literature review and focus groups revealed differences between groups and across environments regarding the experience of being in a crowd. A number of the findings are intuitive, requiring little explanation, including the importance placed on toilet facilities by older individuals.

Analysis of the focus group data indicated that preconceptions of crowd situations strongly affected crowd satisfaction for participants of all ages, supporting previous research (Baum \& Greenberg, 1975; Webb \& Worchel, 1993). On review it was established that two individuals in the healthy adults group were members of the police force. Professional background and its influence on expectations and satisfaction within crowd situations might therefore explain this group raising the issue of violence (members of the police force may have previous experience of dealing with violent crowd situations, affecting their perceptions of crowd satisfaction).

Stress including frustration, anxiety, claustrophobia and vulnerability appeared to impair crowd satisfaction across all groups and crowd situations. Such findings support previous research concerning the negative effects of crowds on stress experienced, suggesting the importance of increasing crowd satisfaction from a health perspective (Cox, Houdmont \& Griffiths, 2006; Evans \& McCoy, 1998; Dion, 1999). However, the positive atmosphere generated during crowd situations was also discussed briefly within all groups and crowd situations, but with a predominance for negative concerns, in line with previous research (Yildirim et al. 2007).

Furthermore, stress appeared to be of primary concern to crowd satisfaction within retail crowding in particular. Such findings emphasize the importance of research into the effects of crowding on retail habits, and the potential benefits of increasing crowd satisfaction, on buyer behavior, supporting previous research (Machleit et al., 2000; Ozdemir, 2008; Whiting \& Nakos, 2008). Furthermore, retail crowding was seen to illicit avoidance, more than other crowd environments. Thus, improved retail crowd satisfaction has potential benefits from a business perspective.
The importance of maintaining control within a crowd, in order to sustain positive feelings, supports previous research (Rodin, Solomon, \& Metcalf, 1978; Worchel \& Yohai, 1979). In addition, based on participants in this study, concern about control was possibly more of an issue for older adults in crowd situations.

Design and organization, and safety and security concerns were found to highly affect crowd satisfaction, irrespective of group differences or crowd environments, highlighting the importance of these issues when considering crowd satisfaction. Moreover, encumbrances featured as a problem for parents of young children in particular, suggesting that when such individuals form the target participation, additional emphasis must be placed on space available for maneuvering encumbrances, to improve crowd satisfaction.

Greater differences were present between groups of individuals, than across crowd situations discussed. This finding could be the result of the methodology used, despite previous research supporting the validity of photographs to represent crowd scenarios (Eroglu \& Machleit, 1990; Ozdemir, 2008). Future research could therefore utilize field based methods to elicit discussion in different crowd environments, enhancing ecological validity.

Insight into factors affecting crowd experience, as revealed in this study, are relevant to individual crowd members and those responsible for generating gatherings. Performance, satisfaction and well-being in such situations should be a concern for both those involved and the organizers, managers and promoters of crowd gatherings. Achieving a positive, high-quality crowd experience for both, is desirable to the overall success of a crowd. Moreover, increased knowledge of crowd behavior could ultimately reduce injuries and fatalities encountered at mass gatherings. Major international disasters include fatalities during pilgrimages to the Hajj in Saudi Arabia (Hughes, 2002; 2003), the Lantern Festival in China (Zhen et al. 2008) and Hillsborough sports stadium in the UK (Smith, 1994). A more systemic approach to understanding crowds should contribute to the avoidance of such incidents. Future research will explore findings further, aiming to develop a more complete model of the factors contributing to crowd experience, their interaction and relative importance.

\section{REFERENCES}

Altman, L. (1975). The environment and social behaviour. California: Brooks Cole: Montererey.

Altman, I. \& Chemers, M. (1980). Culture and Environment, Brooks/Cole: Monterey, CA.

Arbon, P. (2004). The development of conceptual models for mass-gathering health. Prehospital and Disaster Medicine, 19 (3): 208-212.

Baum, A., \& Greenberg, C. I. (1975). Waiting for a crowd: The behavioral and perceptual effects of anticipated crowding. Journal of Personality and Social Psychology, 32(4), 671-679.

Cox, T., Houdmont, J., \& Griffiths, A. (2006). Rail passenger crowding, stress, health and safety in Britain. Transportation Research Part A: Policy and Practice, 40(3), 244-258.

Dion, D. A. (1999). Theoretical and empirical study of retail crowding. European Advances in Consumer Research, 4:1-7.

Eroglu, S. A., \& Machleit, K. A. (1990). An empirical study of retail crowding: Antecedents and consequences. Journal of Retailing, 66(2), 201 .

Evans, G. W., \& McCoy, J. M. (1998). When buildings don't work: the role of architecture in human health. Journal of Environmental Psychology, 18:85-94. 
Evans, G. W., \& Wener, R. E. (2007). Crowding and personal space invasion on the train: Please don't make me sit in the middle. Journal of Environmental Psychology, 27(1), 90-94.

Esser, A. H. (1973). Cottage fourteen: Dominance and territoriality in a group of institutionalized boys. Small Group Behavior, 4,131-146.

Freedman, J. L. (1975). Crowding and behavior: San Francisco: Freeman.

Gérin-Lajoie, M., Richards, C. L., \& McFadyen, B. J. (2005). The negotiation of stationary and moving obstructions during walking: Anticipatory locomotor adaptations and preservation of personal space. Motor Control, 9(3), 242.

Goldenstein, S., Karavelas, M., Metaxas, D., Guibas, L., Aaron, E., \& Goswami, A. (2001). Scalable nonlinear dynamical systems for agent steering and crowd simulation. Computers \& Graphics, 25(6), 983-998.

Haase, R. F., \& Markey, M. J. (1973). A methodological note on the study of personal space. Journal of Consulting and Clinical Psychology, 40(1), 122-125.

Hayduk, L. A. (1983). Personal space: Where we now stand. Psychological Bulletin, 94(2), 293-335

Hughes, R. L. (2002). A continuum theory for the flow of pedestrians. Transportation Research Part B: Methodological, 36(6), 507-535.

Hughes, R. L. (2003). The flow of human crowds. Annual Review of Fluid Mechanics, 35(1), 169-182

Hui, M. K., \& Bateson, J. E. G. (1991). Perceived control and the effects of crowding and consumer choice on the service experience. Journal of Consumer Research, 18(2), 174.

Janchar, T., Samaddar, C., \& Milzman, D. (2000). The mosh pit experience: Emergency medical care for concert injuries. The American Journal of Emergency Medicine, 18(1), 62-63.

Johnson, C. W. (2008). Using evacuation simulations for contingency planning to enhance the security and safety of the 2012 olympic venues. Safety Science, 46(2), 302-322.

Kaya, N., \& Erkip, F. (1999). Invasion of personal space under the condition of short-term crowding: A case study of an automatic teller machine. Journal of Environmental Psychologv, 19(2), 183-189.

Klein, K. \& Harris, B. (1979). Disruptive effects of disconfirmed expectancies about crowding. Journal of Personality and Social Psychology, 37(5), 769-777.

Lee, R. S. C., \& Hughes, R. L. (2007). Minimisation of the risk of trampling in a crowd. Mathematics and Computers in Simulation, 74(1), 29-37.

Machleit, K. A., Eroglu, S. A., \& Mantel, S. P. (2000). Perceived retail crowding and shopping satisfaction: What modifies this relationship? Journal of Consumer Psychology, 9(1), 29-42.

Martinez, V. (2009). Architectural design for space tourism. Acta Astronautica, 64(2-3), 382-390.

Miles, M. B. \& Huberman, A. M. (1994). Qualitative Data Analysis; An Expanded Sourcebook, Second Edition. Sage Publications: London.

Musse, S., \& Thalmann, D. (2001). Hierarchical model for real time simulation of virtual human crowds. IEEE Transactions on Visualization and Computer Graphics, 7(2): 152-164

Ozdemir, A. (2008). Shopping malls: Measuring interpersonal distance under changing conditions and across cultures. Field Methods, 20(3), 226- 248 .

Pons, F., Laroche, M., \& Mourali, M. (2006). Consumer Reactions to Crowded Retail Settings: Cross-Cultural Differences between North America and the Middle East. Psychology \& Marketing, 23(7): $555-572$.

Pons, F., \& Laroche, M. (2007). Cross-cultural differences in crowd assessment. Journal of Business Research, 60(3), 269-276.

Robson, S. K. A. (2008). Scenes from a restaurant: Privacy regulation in stressful situations. Journal of Environmental Psychology, 28(4), 373-378.

Rodin, J., Solomon, S., \& Metcalf, J. (1978). The role of control in mediating perceptions of density. Journal of Personality and Social Psychology, 36. 988-999.

Rüstemli, A. (1992). Crowding effects of density and interpersonal distance. Journal of Social Psychology, 132(1), 51-58.

Schmidt, D. E., \& Keating, J. P. (1979). Human crowding and personal control: An integration of the research. Psychological Bulletin, 86(4), 680-700.

Schopler, I, \& Stockdale, J. (1977). An interference analysis of crowding. Environmental Psychology and Nonverbal Behavior, 1, 81-88.

Sinha, S. P., \& Sinha, S. P. (1991). Personal space and density as factors in task performance and feeling of crowding. Journal of Social Psychology, 131(6), 831-837.
Smith, A., James, C., Jones, R., Langston, P., Lester, E., \& Drury, J. (2009). Modelling contra-flow in crowd dynamics DEM simulation. Safety Science, 47(3), 395-404.

Smith, R. A. (1994). The Hillsborough football disaster: Stress analysis and design codes for crush barriers. Engineering Failure Analysis, I(3), 183-192.

Spacapan, S., \& Cohen, S. (1983). Effects and aftereffects of stressor expectations. Journal of Personality and Social Psychology, 45(6), 1243-1254.

Stokols, D. (1972). On the distinction between density and crowding: Some implications for future research. Psychological Review, 79(3), 275-277.

Tse, A. C. B., Sin, L., \& Yim, F. H. K. (2002). How a crowded restaurant affects consumers' attribution behavior. International Journal of Hospitality Management, 21(4), 449-454

Valach, L., Young, R.A. \& Lynam, M.J. (2002). A Primer for Applied Research in the Social Sciences, Praeger Publishers, Westport, Connecticut

Webb, W. M., \& Worchel, S. (1993). Prior experience and expectation in the context of crowding. Journal of Personality and Social Psychology, 65(3), 512-521.

Whiting, A., \& Nakos, G. (2008). Functional density and its impact on retail satisfaction in cross-cultural contexts: can crowded stores be a good thing for retailers. International Business: Research Teaching and Practice, 2(1).

Worchel, S., \& Teddie, C. (1976). The experience of crowding: A two-factor theory. Journal of Personality and Social Psychology, 34(1), 30-40.

Worchel, S., \& Yohai, S. M. L. (1979). The role of attribution in the experience of crowding. Journal of Experimental Social Psychology, 15(1), 91-104.

Yildirim, K., \& Akalin-Baskaya, A. (2007). Perceived crowding in a café restaurant with different seating densities. Building and Environment, $42(9), 3410-3417$

Zeitz, K. M., Tan, H. M., \& Zeitz, C. J. (2009).Crowd behaviour at mass gatherings: a literature review. Prehospital and Disaster Medicine, 24 (1), $32-8$

Zhang, Q., Liu, M., Wu, C., \& Zhao, G. (2007). A stranded-crowd model (SCM) for performance-based design of stadium egress. Building and Environment, 42(7), 2630-2636.

Zhen, W., Mao, L., \& Yuan, Z. (2008). Analysis of trample disaster and a case study - Mihong Bridge fatality in china in 2004. Safety Science, 46 (8), 1255-1270 